\title{
GMR
}

\section{Epigallocatechin-3-gallate protects retinal vascular endothelial cells from high glucose stress in vitro via the MAPK/ERK-VEGF pathway}

\author{
L. Zhang ${ }^{1}$, Z.K. Zhang ${ }^{1}$ and S. Liang ${ }^{2}$ \\ ${ }^{1}$ Department of Ophthalmology, China-Japan Friendship Hospital, \\ Beijing, China \\ ${ }^{2}$ Worker's Hospital of Handan Steel Factory, Hebei Steel Group, \\ Handan, Hubei, China \\ Corresponding author: Z.K. Zhang \\ E-mail: zzk1986_kx@163.com
}

Genet. Mol. Res. 15 (2): gmr.15027874

Received October 21, 2015

Accepted January 15, 2016

Published June 10, 2016

DOI http://dx.doi.org/10.4238/gmr.15027874

\begin{abstract}
Diabetic retinopathy (DR) is a frequent microvascular complication of diabetes, and one of the most common causes of legal blindness in the world. Epigallocatechin-3-gallate (EGCG) produces an anti-oxidative and anti-inflammatory effect against various human diseases. In this study, we determined the effect of EGCG on a human retinal endothelial cell (HREC) line. The cell viability was determined by a standard MTT assay, while the cell cycle and apoptosis rate were analyzed by flow cytometry. Inflammatory marker expression was detected by enzyme-linked immunosorbent assay. Treatment of HRECs with EGCG $(20$ and $40 \mu \mathrm{M})$ led to a significant decrease in the apoptosis rate $(2.35 \pm 0.56$ and $1.24 \pm 0.32 \%)$. The culture supernatant of cells treated with high glucose concentrations showed significantly higher levels of TNF- $\alpha(598.7 \pm 89.7$ vs $193.2 \pm 38.5 \mathrm{pg} /$ $\mathrm{mL} ; \mathrm{P}<0.001)$, IL-6 (6.16 \pm 0.51 vs $1.61 \pm 0.21 \mathrm{ng} / \mathrm{mL} ; \mathrm{P}<0.001)$,
\end{abstract}


and ICAM-1 (31.6 \pm 4.4 vs $14.8 \pm 2.9 \mathrm{ng} / \mathrm{mL} ; \mathrm{P}<0.001)$ compared to the cells in the control group. EGCG decreased the expression level of phosphorylated p38-mitogen activated protein kinase (MAPK) and extracellular regulated kinase (ERK)1/2. Moreover, EGCG was shown to significantly inhibit the expression of vascular endothelial growth factor (VEGF). Therefore, EGCG treatment ameliorated the negative effect of high glucose concentrations on the cell viability and apoptotic rate. The protective effects of EGCG under high glucose conditions may be attributed to the regulation of inflammatory cytokines and inhibition of the MAPK/ERK-VEGF pathway.

Key words: Diabetic retinopathy; Epigallocatechin-3-gallate; Human retinal endothelial cell

\section{INTRODUCTION}

The past 20 years has seen a major change in the disease spectrum, as well as a significant increase in the prevalence of diabetes mellitus (DM) (Bao et al., 2015). The increase in lifespan of patients is accompanied by a corresponding increase in the incidence of DM-related microvascular complications such as diabetic retinopathy (DR). Additionally, DR is one of the most common causes of legal blindness in the world. Several epidemiological studies have also indicated that DR is a major cause of visual impairment in the elderly in mainland China (Chi et al., 2001).

The pathogenesis of diabetic retinopathy has not been fully clarified (Liu et al., 2014b). The common pathological changes caused by DR include the breakdown of the retinal blood-retinal barrier, retinal neovascularization, macular edema, and retinal detachment (Rangasamy et al., 2014; Liu et al., 2014b). The risk factors for DR include hyperglycemia, polyol metabolism, glycosylation, and diacylglycerol (Safi et al., 2014). Oxidative stress, an increase in free radical content, inflammation, and cytokine production, has also been shown to produce a significant effect on the pathogenesis of DR (Rosales et al., 2014; Tan and de Haan, 2014). Inflammation is one of the key pathogeneses of DR (Ma et al., 2014); in fact, a reduction in inflammation has been shown to exert a positive effect on the development and progression of DR. However, the use of active anti-inflammatory agents causes multiple adverse effects, and therefore cannot be used in a majority of the patients. This highlights the need for the development of therapeutic strategies to effectively inhibit inflammation in DR.

Green tea consumption has been reported to prevent the development of various diseases, including cardiovascular disease, DM, hypertension, and several types of cancers (Wang et al., 2010b; Khan and Mukhtar, 2013; Ma et al., 2014). Additionally, some studies have reported the protective effects of green tea against the common side effects of DM. Epigallocatechin-3-gallate (EGCG; chemical structure shown in Figure 1) is a polyphenol that has been shown to exert anti-oxidative, anti-inflammatory, and anti-atherogenic effects in both in vitro and in vivo studies (Hossain et al., 2014). The results of a previous study conducted to detect the neuroprotective effects of EGCG from green tea on diabetic retina and retinal cells cultured under diabetic conditions (Lee et al., 2014) revealed complete restoration of the glial fibrillary acidic protein (GFAP), oxidative retinal markers, and glutamine synthetase levels. 
Therefore, it was theorized that green tea might protect the retina against glutamate toxicity via an antioxidant mechanism.

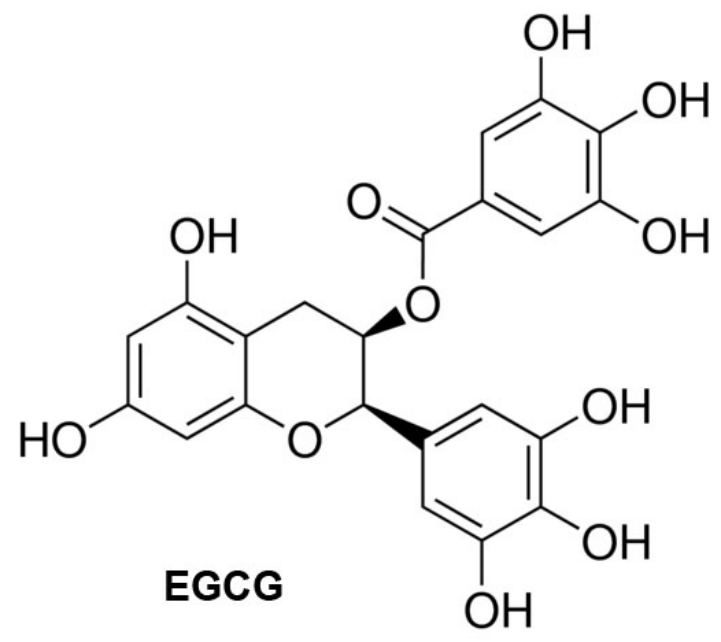

Figure 1. Chemical structure of EGCG.

EGCG has been previously reported to produce anti-inflammatory effects in several diseases (Cao et al., 2014; Wu et al., 2014); moreover, inflammation has been shown to significantly promote the development of DR. Therefore, the detection of the effects of EGCG on inflammation in retina under high glucose conditions is of paramount importance. In this study, we have attempted to describe a novel mechanism by which EGCG protects the retina under high glucose conditions, in order to detect the therapeutic potential of EGCG in the treatment of DR. Here, we have assumed that EGCG inhibits high glucose-induced apoptosis and inflammation in retinal microvascular cells. Additionally, we have described the potential role of the mitogen-activated protein kinase/extracellular signal-regulated kinase-vascular endothelial growth factor (MAPK/ERK-VEGF) pathway in the protective effect of EGCG.

\section{MATERIAL AND METHODS}

\section{Cell culture}

A human retinal endothelial cell (HREC) line was purchased from Kebai Biologic Technologies (Jiangsu, China). The cells were cultured in Dulbecco's modified Eagle's medium (DMEM; Gibco, Grand Island, NY, USA) supplemented with 10\% fetal bovine serum (Sigma-Aldrich, St. Louis, MO, USA) and a 1\% antibiotic/antimycotic solution containing 1 x $10^{4} \mathrm{U}$ penicillin and $10 \mathrm{mg}$ streptomycin at $37^{\circ} \mathrm{C}$ in a humidified atmosphere of $5 \% \mathrm{CO}_{2}$ and $95 \%$ air. Cells passaged 6 to 9 times were used in this study.

\section{EGCG treatment}

The cells supplemented with glucose at normal $(1 \mathrm{~g} / \mathrm{L})$ or high $(4.5 \mathrm{~g} / \mathrm{L})$ levels; 
subsequently, the cells were stimulated with EGCG (purity > 98\%; Sigma-Aldrich) for $6 \mathrm{~h}$. The treated cells and clear supernatant were used in cell viability studies, as well as western blot, flow cytometry, and enzyme-linked immunosorbent assay (ELISA).

\section{Cell viability}

The cell viability was determined using a standard MTT 3-(4,5-dimethylthiazol-2yl)-2,5-diphenyltetrazolium (Sigma-Aldrich) assay. The cultured HRECs were treated with $0.5 \mathrm{mg} / \mathrm{mL}$ MTT for $6 \mathrm{~h}$ in the dark at $37^{\circ} \mathrm{C}$. The absorbance was determined at $570 \mathrm{~nm}$ using an automated microplate reader (Spectra Max 340; Molecular Devices, Sunnyvale, CA, USA) after washing the cells and allowing the produced formazan salts to be dissolved. All experiments were performed in triplicate, and the mean values were used for data analysis.

\section{Cell cycle analysis}

The cell cycle was analyzed using a flow cytometry-based method. The cultured HRECs were digested with pancreatin, collected, and subsequently washed with cold PBS. The cells were fixed in $70 \%$ cold alcohol for $2 \mathrm{~h}$ at $4^{\circ} \mathrm{C}$. The fixed cells were resuspended and subsequently centrifuged at $1000 \mathrm{~g}$ for $5 \mathrm{~min}$. The pelleted cells were washed twice with cold PBS and subsequently analyzed. The cells were treated with their corresponding propidium iodide (PI)-labeled antibodies, $100 \mu \mathrm{g} / \mathrm{mL}$ RNase A, and $0.2 \%$ Triton X-100 in the dark for $2 \mathrm{~h}$. Percentages of the cells in different phases of the cell cycle were determined using the ModFitLT software (Verity Software House Inc., Topsham, ME, USA). Each independent experiment required at least $20 \times 10^{3}$ cells; all experiments were performed in triplicate.

\section{Apoptosis analysis}

Cell apoptosis was analyzed by flow cytometry using the apoptotic and cell death probes Annexin V and propidium iodide (PI), respectively. Apoptosis was detected in the HRECs using a standard Apoptosis detection kit (Vazyme Biotech Co., Ltd., Piscataway, NJ, USA). HRECs in each group were centrifuged to remove the culture medium, washed with PBS, and stained with Annexin V-fluorescein isothiocyanate (FITC) and PI in binding buffer at room temperature for $5 \mathrm{~min}$. The cells were then detected by flow cytometry and fluorescence microscopy within $1 \mathrm{~h}$ at an excitation wavelength of $488 \mathrm{~nm}$. The green and red fluorescence of FITC and PI were detected by the FL1 and FL2 channels, respectively. The results were analyzed using the CellQuest software (FACSCalibur; BD Biosciences, Franklin Lakes, NJ, USA).

\section{Western-blot analysis}

HRECs were washed twice with cold PBS and subsequently lysed by incubating with ice-cold RIPA lysis buffer (P0013C; Beyotime, Shanghai, China) for $5 \mathrm{~min}$. The lysates were scraped off the plates and centrifuged at $1.4 \times 10^{5} \mathrm{~g}$ for $20 \mathrm{~min}$ at $4{ }^{\circ} \mathrm{C}$. The proteins in the supernatant were quantified using a standard BCA kit (ThermoFisher Scientific, Waltham, MA, USA). Thirty micrograms of the protein were loaded for all further analyses. The nitrocellulose (NC) membrane was blocked with a $20 \mathrm{mM}$ TBS buffer containing $5 \%$ non-fat 
dry milk and $1 \%$ Tween-20 $(\mathrm{pH} 7.6)$ for $1 \mathrm{~h}$ at room temperature. The proteins were separated by SDS-PAGE and electro-transferred to the NC membrane (Optitran; GE Healthcare, Little Chalfont, UK). The membrane was blocked with the blocking buffer, washed, and subsequently incubated with primary antibodies against p38 MAPK, phospho-p38 MAPK (p-p38 MAPK), ERK1/2, p-ERK1/2 (1:1000; Cell Signaling Technologies, Boston, MA, USA), and VEGF (1:1000; Santa Cruz Biotechnology, Santa Cruz, CA, USA) overnight. The membrane was then incubated with a horseradish peroxidase-conjugated secondary antibody for $1 \mathrm{~h}$ at room temperature. Anti-actin antibodies (Santa Cruz Biotechnology) were used for normalization of the data. The signals were detected and analyzed using the ImageJ software (National Institute of Health, Bethesda, MD, USA).

\section{ELISA for inflammatory marker detection}

The expression of inflammatory markers was detected using a standard ELISA kit (Sigma-Aldrich) in accordance with the manufacturer protocols. The cell culture supernatant from each group of HRECs was subjected to ELISA testing to detect the expression of tumor necrosis factor- $\alpha$ (TNF- $\alpha$ ), interleukin-6 (IL-6), and intercellular cell adhesion molecule-1 (ICAM-1). All assays were performed in triplicate.

\section{Statistical analyses}

All results are reported as means \pm standard deviation (SD) of three independent experiments. The differences in continuous variables between each group were assessed by an independent $t$-test. Differences between more than three groups were determined by oneway analysis of variance (ANOVA). All data analyses were conducted using the SPSS v.13.0 software package (SPSS Inc., Chicago, IL, USA). The differences were considered to be statistically significant at $\mathrm{P}$ values $<0.05$.

\section{RESULTS}

\section{Effect of EGCG on HREC viability under high glucose conditions}

We first attempted to determine the time dependency of the effect of high glucose conditions on HREC viability (Figure 2A). Treatment of HRECs with $4.5 \mathrm{~g} / \mathrm{L}$ glucose for 6 $\mathrm{h}$ did not produce a significant difference in the cell viability compared to the control cells. However, we observed a significant decrease in cell viability with the increase in duration of treatment: incubation of HRECs with a high glucose concentration for 12, $24(0.800 \pm 0.050)$, and $48(0.757 \pm 0.061) \mathrm{h}$ led to a significant corresponding decrease in cell viability $(\mathrm{P}<0.01)$. The 48-h incubation period was used in all future experiments, as a significant and stable decrease in cell viability was observed at this time point.

The effect of EGCG treatment on high glucose-pretreated HRECs was investigated by incubating cells cultured in serum-free DMEM at $70 \%$ confluence with increasing concentrations of EGCG for $24 \mathrm{~h}$. Treatment with $10 \mu \mathrm{M}$ EGCG did not significantly protect the HRECs against unfavorable glucose conditions. However, treatment of high glucosepretreated cells with higher concentrations of EGCG $(20 \mu \mathrm{M}, \mathrm{P}<0.001 ; 40 \mu \mathrm{M}, \mathrm{P}<0.001)$ 
exerted a significant protective effect on the cells, leading to an increase in cell viability (Figure 2B).
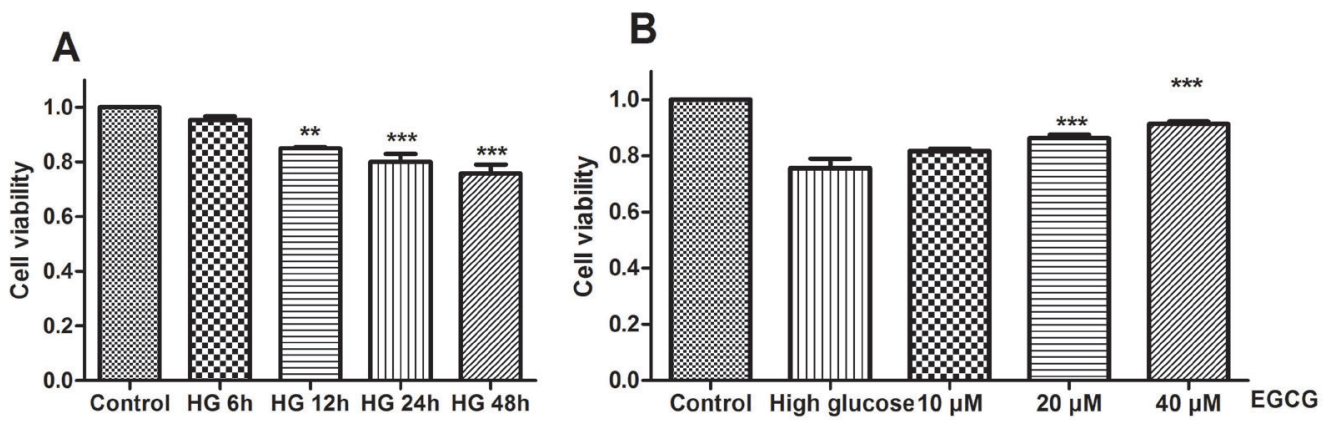

Figure 2. Effect of EGCG on human retinal endothelial cell (HREC) viability under high glucose conditions. A. Effect of high glucose concentrations on HREC viability at different time points. B. HRECs pretreated with high glucose were treated with EGCG. Cell viability detected using the MTT assay. All values are means \pm SD of three experiments; $\mathrm{N}=6$.

\section{Protective effect of EGCG against apoptosis in HRECs}

Apoptosis occurs as a result of abnormalities in various pathological processes, such as the decrease in cell viability induced by high glucose conditions. The possible protective effect of EGCG against apoptosis in HRECs (induced by high glucose concentrations) was investigated in this study. HRECs treated with high glucose concentrations for $48 \mathrm{~h}$ showed a significantly higher apoptosis rate than the control cells treated with a normal amount of glucose $(5.52 \pm 0.82$ vs $1.10 \pm 0.21 \%, \mathrm{P}<0.001)$. However, treatment of HRECs (cultured under high glucose conditions) with EGCG $(20$ and $40 \mu \mathrm{M})$ for $24 \mathrm{~h}$ led to a significant decrease in the apoptosis rate $(2.35 \pm 0.56$ and $1.24 \pm 0.32 \%$, respectively) in a dose-dependent manner; that is, EGCG exerted a dose-dependent protective effect against apoptosis in HRECs cultured under high glucose conditions (Figure 3).

\section{EGCG modifies the cell cycle distribution of HRECs cultured under high glucose conditions}

Changes in the cell cycle produce a significant effect on high glucose-related stress. Here, we attempted to determine if the protective effect of EGCG was caused as a result of the cell being arrested at a specific point in the cell cycle via flow cytometry. This analysis revealed that HRECs treated with high glucose showed an accelerated G0/G1 phase and decelerated $\mathrm{S}$ phase (Table 1). Treatment with different concentrations of EGCG caused a significant decrease and increase in the percentage of cells in the G0/G1 and S phase, respectively.

\section{EGCG suppressed the MAPK/ERK-VEGF pathway in HRECs induced with high glucose}

The MAPK/ERK pathway has been shown to play a role in a number of inflammatory 
processes, which play key roles in the development of DC. Therefore, we attempted to detect the anti-inflammatory effect of EGCG under high glucose conditions. We observed no significant differences in the p38-MAPK and MERK1/2 levels between the high glucose and control groups. However, p-p38-MAPK and p-MERK1/2 levels significantly increased under high glucose conditions, which were significantly decreased by EGCG treatment. On the other hand, the p38-MAPK and MERK1/2 levels were not significantly affected by the EGCG treatment. Thus, it could be inferred that EGCG exerted a significant anti-inflammatory effect by suppressing the phosphorylation mechanism in the MAPK/ERK pathway induced by high glucose.
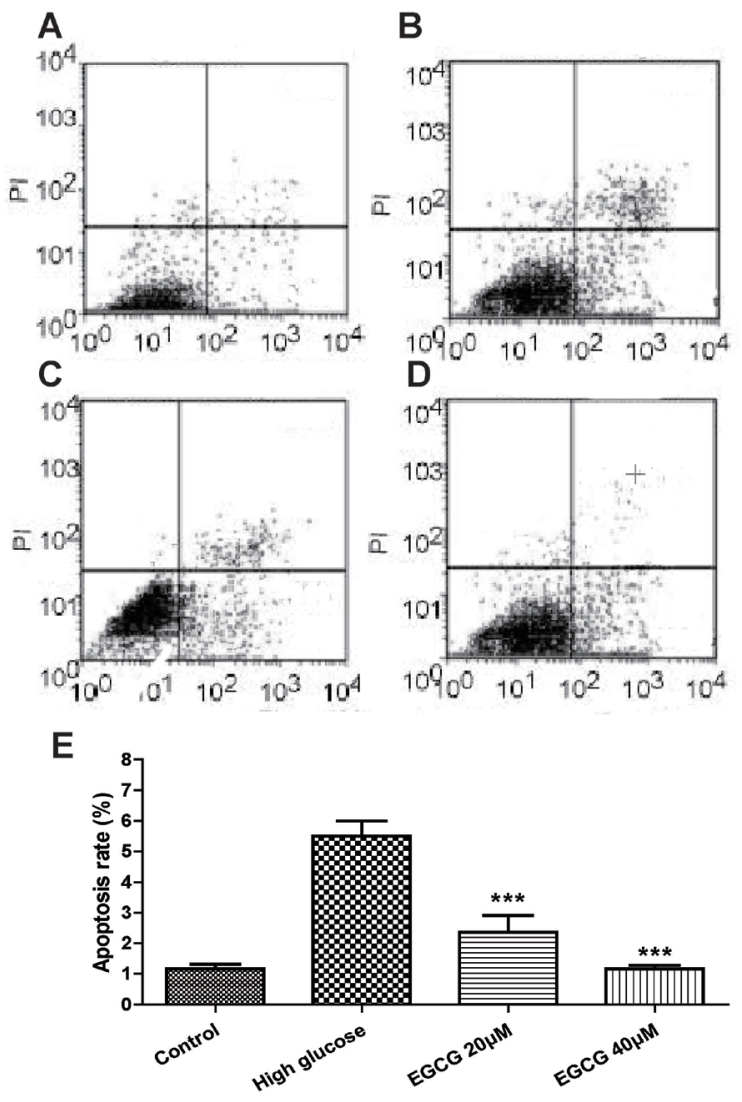

Figure 3. EGCG modulates high glucose-induced oxidative stress in HRECs. A. Control group; B. high glucose group; C. $20 \mu \mathrm{M}$ EGCG-treated group; D. $20 \mu \mathrm{M}$ astaxanthin-treated group; E. oxidative rate in each group.

Table 1. Effect of EGCG on the cell cycle distribution in high-glucose treated HRECs.

\begin{tabular}{l|c|c|c}
\hline Cell cycle & G0/G1 & S & G2/M \\
\hline Control & $70.2 \pm 7.9 \%$ & $15.6 \pm 3.5 \%$ & $14.2 \pm 2.9 \%$ \\
\hline High glucose & $79.0 \pm 8.4 \%$ & $8.9 \pm 2.2 \%$ & $12.1 \pm 3.5 \%$ \\
\hline High glucose + $20 \mu \mathrm{M}$ EGCG & $73.1 \pm 8.6 \%$ & $13.5 \pm 4.0 \%$ & $13.4 \pm 3.8 \%$ \\
\hline High glucose $+40 \mu \mathrm{M}$ EGCG & $73.3 \pm 8.9 \%$ & $13.6 \pm 3.5 \%$ & $13.1 \pm 3.2 \%$ \\
\hline P value & 0.033 & 0.041 & 0.288 \\
\hline
\end{tabular}


VEGF is one of the most important growth factors affecting DR progression. VEGF has also been shown to play a role in inflammation in various pathological processes. Westernblot analysis of the different HREC groups revealed that high glucose concentrations induced a significant increase in VEGF expression. Alternately, EGCG was shown to induce a significant reduction in the expression VEGF in a dose-dependent manner (Figure 4).

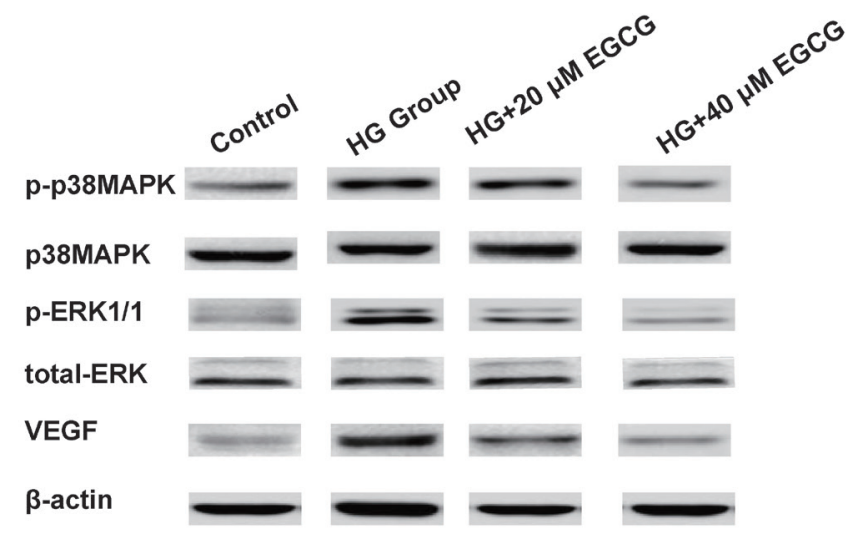

Figure 4. Effect of EGCG on the MAPK/ERK-VEGF pathway in high glucose-induced HRECs. The expression of p38 MAPK, p-p38 MAPK, MERK1/2, p-MERK1/2, and VEGF in each group was determined.

\section{Effect of EGCG on inflammatory marker expression}

The TNF- $\alpha$, IL-6, and ICAM-1 levels in the culture supernatant were determined by standard ELISA. The culture supernatant of cells treated with high glucose concentrations showed significantly higher levels of TNF- $\alpha(598.7 \pm 89.7 v s 193.2 \pm 38.5 \mathrm{pg} / \mathrm{mL} ; \mathrm{P}<0.001)$, IL-6 (6.16 \pm 0.51 vs $1.61 \pm 0.21 \mathrm{ng} / \mathrm{mL} ; \mathrm{P}<0.001)$, and ICAM-1 $(31.6 \pm 4.4 v s 14.8 \pm 2.9 \mathrm{ng} / \mathrm{mL}$; $\mathrm{P}<0.001)$ compared to the cells in the control group. On the other hand, treatment with 20 or 40 $\mu \mathrm{M}$ EGCG significantly inhibited this increase in TNF- $\alpha$, IL-6, and ICAM-1 levels (Figure 5).

A

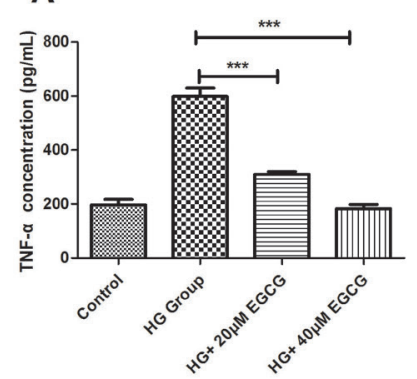

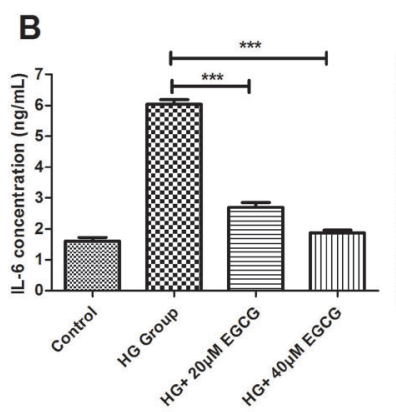

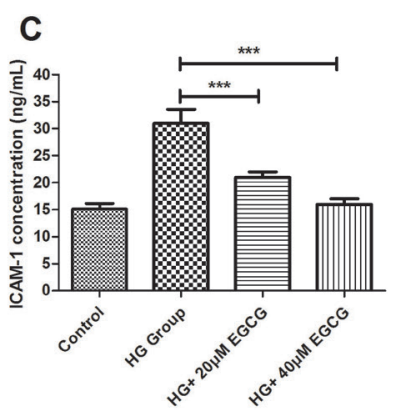

EGCG

Figure 5. Effect of EGCG on the inflammatory biomarkers in HRECs cultured under high glucose conditions. Expression of (A) tumor necrosis factor (TNF)- $\alpha,(\mathbf{B})$ interleukin (IL)-6, and (C) ICAM-1 in the control, high glucose, and $20 \mu \mathrm{M}$ EGCG- and $40 \mu \mathrm{M}$ EGCG-treated groups. 


\section{DISCUSSION}

DR is one of the most common and severe microvascular complications of diabetes mellitus (Tong et al., 2008). In this study, we showed that EGCG significantly improved the cell viability of HRECs cultured under high glucose conditions. EGCG was also shown to exert a protective effect against high glucose-induced apoptosis in HRECs. Moreover, we observed that EGCG reduced the expression of inflammatory markers such as TNF- $\alpha$, IL-6, and ICAM-1 in the HREC culture supernatant, as well as the levels of phosphorylated p38MAPK and ERK1/2. However, we observed no significant changes in the levels of p38-MAPK and ERK1/2. Additionally, EGCG significantly inhibited the expression of VEGF. These results indicated that EGCG ameliorated the negative effects of high glucose concentrations on cell viability and apoptosis. The protective effects of EGCG under high glucose conditions were attributed to the regulation of inflammatory cytokines and inhibition of the MAPK/ERKVEGF pathway.

DR is a major cause of vision loss in adults (Ellis et al., 2013). A number of studies have reported a positive correlation between inflammation and DR and its progression. Therefore, a number of researchers have attempted to devise methods to control the inflammatory reaction as a novel therapeutic measure. Recent studies have suggested that EGCG significantly improves the pathology of various diseases, including metabolism dysfunction, cardiovascular disease, and cancer (Jiang et al., 2014). EGCG is natural anti-inflammatory agent that has been reported to resist various inflammatory processes. For example, Liu et al. (2014a) discovered that EGCG significantly inhibited the release of TNF- $\alpha$, interferon (IFN)- $\gamma$, IL-4, and IL-6 in the serum and reduced the level of malondialdehyde (MDA) in concanavalin A-induced hepatic injury in mice, suggesting that EGCG protects against liver injury via its anti-inflammatory and anti-oxidant activity.

Interestingly, EGCG was found to increase the phosphorylation of the MAPK/ERK pathway, rather than the expression of the total MAPK/ERK pathway content. Moreover, we observed a significant association between this activation and the protective effects of EGCG. Previous studies have reported that the MERK/ERK pathway plays a key role in the development of DR by upregulating the chronic inflammation in DR. Moreover, IL-1 $\beta$ was shown to upregulate the IL-8 production in human Müller cells via the activation of the p38 MAPK and ERK1/2 signaling pathways (Liu et al., 2014c). This result showed that an increase in IL-8 expression was accompanied by a significant increase in the phosphorylation of p38MAPK and -ERK. These data are in agreement with our findings regarding the phosphorylation of the MAPK/ERK pathway in the presence of high glucose concentrations. Here, EGCG was shown to reduce the phosphorylation of p38 MAPK and ERK in a dose-dependent manner in HRECs treated with high glucose concentrations. We also surmised that the protective effect of EGCG might function through the suppression of a key downstream factor of the MAPK/ ERK pathway - VEGF.

EGCG was shown to significantly inhibit the expression of VEGF in a dose-dependent manner. Previous reports have suggested that blocking of the VEGF receptor signaling pathway can completely prevent the formation of retinal neovascularization, which indicated that VEGF plays a vital role in the formation of new blood vessels (Wang et al., 2010a). DR can be induced by the injection of VEGF in normal animals, suggesting the significant role played by VEGF during the early stages of DR (Gupta et al., 2013). Chronic inflammation is 
known to play a major role in the development of DR from DM. In fact, chronic inflammation has been considered as the key pathology of VEGF secretion and angiogenesis, with VEGF stimulating the inflammatory condition. The results of this study showed that EGCG treatment caused a significant decrease in the VEGF expression compared to that seen in the control group. This was attributed to the inhibition of VEGF in the retina, causing a reduction in the proliferation of blood vessels and retinal thickening.

In conclusion, EGCG treatment has been shown to protect HRECs against the negative effects of high glucose conditions through the inhibition of the MAPK/ERK pathway. VEGF is a key downstream factor of the MAPK/ERK pathway that was significantly regulated by EGCG. Moreover, the expression of inflammatory markers such as TNF- $\alpha$, IL-6, and ICAM-1 were also decreased by EGCG. Therefore, EGCG may be used to prevent and treat DR. The results of this study must be validated by further in vivo studies.

\section{Conflicts of interest}

The authors declare no conflict of interest.

\section{REFERENCES}

Bao W, Yeung E, Tobias DK, Hu FB, et al. (2015). Long-term risk of type 2 diabetes mellitus in relation to BMI and weight change among women with a history of gestational diabetes mellitus: a prospective cohort study. Diabetologia 58: 1212-1219. http://dx.doi.org/10.1007/s00125-015-3537-4

Cao Y, Bao S, Yang W, Zhang J, et al. (2014). Epigallocatechin gallate prevents inflammation by reducing macrophage infiltration and inhibiting tumor necrosis factor- $\alpha$ signaling in the pancreas of rats on a high-fat diet. Nutr. Res. 34: 1066-1074. http://dx.doi.org/10.1016/j.nutres.2014.10.004

Chi ZS, Lee ET, Lu M, Keen H, et al. (2001). Vascular disease prevalence in diabetic patients in China: standardised comparison with the 14 centres in the WHO Multinational Study of Vascular Disease in Diabetes. Diabetologia 44 (Suppl 2): S82-S86. http://dx.doi.org/10.1007/PL00002944

Ellis D, Burgess PI and Kayange P (2013). Management of diabetic retinopathy. Malawi Med. J. 25: 116-120.

Gupta N, Mansoor S, Sharma A, Sapkal A, et al. (2013). Diabetic retinopathy and VEGF. Open Ophthalmol. J. 7: 4-10. http://dx.doi.org/10.2174/1874364101307010004

Hossain MZ, Patel K and Kern SE (2014). Salivary $\alpha$-amylase, serum albumin, and myoglobin protect against DNAdamaging activities of ingested dietary agents in vitro. Food Chem. Toxicol. 70: 114-119. http://dx.doi.org/10.1016/j. fct.2014.05.002

Jiang L, Tao C, He A and He X (2014). Overexpression of miR-126 sensitizes osteosarcoma cells to apoptosis induced by epigallocatechin-3-gallate. World J. Surg. Oncol. 12: 383. http://dx.doi.org/10.1186/1477-7819-12-383

Khan N and Mukhtar H (2013). Tea and health: studies in humans. Curr. Pharm. Des. 19: 6141-6147. http://dx.doi. org/10.2174/1381612811319340008

Lee HS, Jun JH, Jung EH, Koo BA, et al. (2014). Epigalloccatechin-3-gallate inhibits ocular neovascularization and vascular permeability in human retinal pigment epithelial and human retinal microvascular endothelial cells via suppression of MMP-9 and VEGF activation. Molecules 19: 12150-12172. http://dx.doi.org/10.3390/molecules 190812150

Liu D, Zhang X, Jiang L, Guo Y, et al. (2014a). Epigallocatechin-3-gallate (EGCG) attenuates concanavalin A-induced hepatic injury in mice. Acta Histochem. 116: 654-662. http://dx.doi.org/10.1016/j.acthis.2013.12.002

Liu MM, Wolfson Y, Bressler SB, Do DV, et al. (2014b). Comparison of time- and spectral-domain optical coherence tomography in management of diabetic macular edema. Invest. Ophthalmol. Vis. Sci. 55: 1370-1377. http://dx.doi. org/10.1167/iovs.13-13049

Liu X, Ye F, Xiong H, Hu D, et al. (2014c). IL-1 beta upregulates IL-8 production in human Muller cells through activation of the p38 MAPK and ERK1/2 signaling pathways. Inflammation 37: 1486-1495. http://dx.doi.org/10.1007/s10753014-9874-5

Ma K, Xu Y, Wang C, Li N, et al. (2014). A cross talk between class A scavenger receptor and receptor for advanced glycation end-products contributes to diabetic retinopathy. Am. J. Physiol. Endocrinol. Metab. 307: E1153-E1165. 
http://dx.doi.org/10.1152/ajpendo.00378.2014

Rangasamy S, McGuire PG, Franco Nitta C, Monickaraj F, et al. (2014). Chemokine mediated monocyte trafficking into the retina: role of inflammation in alteration of the blood-retinal barrier in diabetic retinopathy. PLoS One 9: e108508. http://dx.doi.org/10.1371/journal.pone. 0108508

Rosales MA, Silva KC, Duarte DA, de Oliveira MG, et al. (2014). S-nitrosoglutathione inhibits inducible nitric oxide synthase upregulation by redox posttranslational modification in experimental diabetic retinopathy. Invest. Ophthalmol. Vis. Sci. 55: 2921-2932. http://dx.doi.org/10.1167/iovs.13-13762

Safi SZ, Qvist R, Kumar S, Batumalaie K, et al. (2014). Molecular mechanisms of diabetic retinopathy, general preventive strategies, and novel therapeutic targets. BioMed Res. Int. 2014: 801269. http://dx.doi.org/10.1155/2014/801269

Tan SM and de Haan JB (2014). Combating oxidative stress in diabetic complications with Nrf2 activators: how much is too much? Redox Rep. 19: 107-117. http://dx.doi.org/10.1179/1351000214Y.0000000087

Tong Z, Yang Z, Patel S, Chen H, et al.; Genetics of Diabetes and Diabetic Complication Study Group (2008). Promoter polymorphism of the erythropoietin gene in severe diabetic eye and kidney complications. Proc. Natl. Acad. Sci. USA 105: 6998-7003. http://dx.doi.org/10.1073/pnas.0800454105

Wang F, Li HM, Wang HP, Ma JL, et al. (2010a). siRNA-mediated knockdown of VEGF-A, VEGF-C and VEGFR-3 suppresses the growth and metastasis of mouse bladder carcinoma in vivo. Exp. Ther. Med. 1: 899-904.

Wang QM, Gong QY, Yan JJ, Zhu J, et al. (2010b). Association between green tea intake and coronary artery disease in a Chinese population. Circ. J. 74: 294-300. http://dx.doi.org/10.1253/circj.CJ-09-0543

Wu J, Xu X, Li Y, Kou J, et al. (2014). Quercetin, luteolin and epigallocatechin gallate alleviate TXNIP and NLRP3mediated inflammation and apoptosis with regulation of AMPK in endothelial cells. Eur. J. Pharmacol. 745: 59-68. http://dx.doi.org/10.1016/j.ejphar.2014.09.046 